Alex Mendonça Bimbato

alexbimbato@unifei.edu.br

\author{
Luiz Antonio Alcântara Pereira \\ Member, $A B C M$ \\ luizantp@unifei.edu.br \\ UNIFEI \\ Instituto de Engenharia Mecânica \\ 37500-903 Itajubá, MG, Brazil \\ Miguel Hiroo Hirata \\ Emeritus Member, ABCM \\ hirata@superonda.com.br \\ FAT/UERJ \\ Campos Regional de Resende \\ Resende, RJ, Brazil
}

\section{Simulation of Viscous Flow around a Circular Cylinder near a Moving Ground}

The objective of this paper is to study the vortex shedding from a circular cylinder near a moving ground; this is done using the Vortex Method. A moving ground has been widely used in the field of experimental vehicle aerodynamics, especially of high-performance racing cars, to properly consider the ground effect on the vehicles aerodynamic. In experimental work as well as in numerical simulations, the ground plane develops a boundary layer that interferes with the body viscous wake, leading to not so precise results. A ground moving with the incoming flow velocity, however, does not allow the development of a boundary layer. The results of our numerical simulations show that the critical drag behavior is directly related to a global change in the wake structure of the cylinder in the ground effect. Comparisons with experimental data are encouraging. Keywords: moving ground, near wake structure, aerodynamic loads, vortex method

\section{Introduction}

The flow around circular cylinders has been extensively studied due to its importance in many practical applications, such as heat exchangers, chimneys and off-shore platforms. In scientific terms, the flow around circular cylinders includes a variety of fluid dynamics phenomena, such as separation, vortex shedding and the transition to turbulence. The mechanisms of vortex shedding and its suppression have significant effects on the various fluid-mechanical properties of practical interest: flow-induced forces, vibrations and noises, and the efficiencies of heat and mass transfer, for example. Cylinders having a two-dimensional structure are very suitable for restricting the complexity and thus observing the fundamental features of the flow.

The fluid flow around a circular cylinder close to a plane wall is governed not only by the Reynolds number but also by the gap between the cylinder and the ground, h, characterized by the gap ratio $\mathrm{h} / \mathrm{d}$ (d is cylinder diameter). The fundamental effects of gap ratio have been observed by Taneda (1965), Roshko et al. (1975), Bearman and Zdravkovich (1978), Burest and Lanciotti (1979), Angrilli et al. (1982), Grass et al. (1984), Zdravkovich (1985a), Price et al. (2002) and Lin et al. (2005).

The influence of the boundary layer that develops on the ground and interferes with the body viscous wake is complex and is still unclear despite several intensive studies reported so far. Roshko et al. (1975) measured the time-averaged drag and lift coefficients, $C_{D}$ and $\mathrm{C}_{\mathrm{L}}$, for a circular cylinder placed near a fixed wall in a wind tunnel at $\operatorname{Re}=2.0 \times 10^{4}$, which lies in the upper-subcritical flow regime; they showed that $C_{D}$ rapidly decreased and $C_{L}$ increased as the cylinder came close to the wall. Zdravkovich (1985b) observed, in his force measurements performed at $4.8 \times 10^{4}<\mathrm{Re}<3.0 \times 10^{5}$, that the rapid decrease in drag occurred as the gap was reduced to less than the thickness of the boundary layer $\delta / d$ on the ground, and concluded that the variation of $C_{D}$ was dominated by $h / \delta$ rather than by the conventional gap ratio $\mathrm{h} / \mathrm{d}$. He also noted that the $\mathrm{C}_{\mathrm{L}}$ could be significantly affected by the state of the boundary layer, although it was insensitive to the thickness of the boundary layer.

Zdravkovich (2003) reported the drag behavior for circular cylinder placed near a ground running at the same speed as the freestream for higher Reynolds number of $2.5 \times 10^{5}$, which lies within the critical flow regime rather than the subcritical flow regime. The experiment by Zdravkovich (2003) showed some differences to all the above studies. First, practically no boundary layer was developed on the ground. Second, the decrease in drag due to the decrease in $\mathrm{h} / \mathrm{d}$ did not occur in his measurements. The differences found were attributed to the non-existence of the wall boundary layer or the higher Reynolds number that seems to be within the critical flow regime rather than within the sub-critical flow regime, or any other influencing factors.

Nishino (2007) presented experimental results of a circular cylinder with an aspect ratio of 8.33, with and without end-plates, placed near and parallel to a ground running at the same speed as the freestream; on the ground surface almost none boundary layer development was observed. Measurements were carried out at two upper-subcritical Reynolds numbers of 0.4 and $1.0 \times 10^{5}$. The results produced new insights into the physics of the phenomena. According to experiments for the cylinder with end-plates on which the oil flow patterns were observed to be essentially twodimensional the drag rapidly decreases as $\mathrm{h} / \mathrm{d}$ decreases to less than 1.0 , but become constant for $\mathrm{h} / \mathrm{d}$ less than 0.85 , unlike the usually observed results obtained with a fixed ground (as will be plotted later in Fig. 6(a)).

This paper describes a mesh-free method used to calculate global as well as local quantities of a high Reynolds number flow around a circular cylinder located near a moving ground. The twodimensional aerodynamic characteristics are investigated at a Reynolds number of $1.0 \times 10^{5}$; due to this fact, even with such a high Reynolds number value, no attempt for turbulence modeling were made once these aspects have a strong three-dimensional component; see Alcântara Pereira et al. (2002). We use the Vortex Method to analyze the influence of the ground on the flow and force characteristics; with a ground running at the same speed as the freestream, no shear layer develops on its surface and, therefore, no vorticity generation is necessary except on the cylinder surface. Comparisons are made with experimental results presented by Nishino (2007).

Vortex Methods have been developed and applied for the analysis of complex, unsteady and vortical flows, because they consist of simple algorithm based on physics of flow (Kamemoto, 2004). Important features of the Vortex Method (Chorin, 1973; Leonard, 1980; Sarpakaya, 1989; Lewis, 1999; Kamemoto, 2004; Alcântara Pereira et al., 2004; Stock 2007) are:

(i) It is a numerical technique suitable for the solution of convection/diffusion type equations like the Navier-Stokes ones;

(ii) It is a suitable technique for direct simulation and largeeddy simulation;

Paper accepted April, 2009. Technical Editor: Francisco R. Cunha 
(iii) It is a mesh free technique; the vorticity field is represented by a cloud of discrete free vortices that move with the fluid velocity.

Vortex cloud simulation offers a number of advantages over the more traditional Eulerian schemes for the analysis of the external flow that develops in a large domain; the main reasons are:

(i) As a fully mesh-less scheme, no grid is necessary;

(ii) The computational efforts are directed only to the regions with non-zero vorticity and not to all the domain points as it is done in the Eulerian formulations;

(iii) The far away downstream boundary condition is taken care automatically, which is relevant for the simulation of the flow around a bluff body (or an oscillating body) that has a wide viscous wake.

\section{Nomenclature}

$$
\begin{aligned}
\overline{\mathrm{A}}_{\mathrm{CL}} & =\text { Mean lift coefficient amplitude } \\
C_{D} & =\text { Drag coefficient } \\
C_{L} & =\text { Lift coefficient } \\
\mathrm{C}_{\mathrm{P}} & =\text { Pressure coefficient } \\
\mathrm{d} & =\text { Cylinder diameter } \\
\mathrm{f} & =\text { Vortex shedding frequency } \\
\mathbf{G} & =\text { Green's function } \\
\mathrm{h} & =\text { Gap between the cylinder and the ground } \\
\mathbf{K} & =\text { Biot-Savart kernell } \\
\mathrm{n} & =\text { Coordinate normal to solid surface } \\
\mathrm{p} & =\text { Pressure field } \\
\mathrm{Re} & =\text { Reynolds number } \\
\mathrm{S} & =\text { Domain boundary } \\
\mathrm{S}_{1} & =\text { Body surface } \\
\mathrm{S}_{2} & =\text { Ground surface } \\
\mathrm{S}_{\infty} & =\text { Far away boundary } \\
\mathrm{S}_{\mathrm{t}} & =\text { Strouhal number } \\
\mathrm{U} & =\text { Uniform incoming flow }
\end{aligned}
$$

$$
\begin{aligned}
\mathbf{u} & =\text { Velocity field } \\
\mathbf{u i} & =\text { Velocity induced } \text { by the incident flow } \\
\mathbf{u b} & =\text { Velocity induced } \text { by the solid surfaces } \\
\mathbf{u v} & =\text { Velocity induced by the vortex cloud } \\
\overline{\mathrm{Y}} & =\text { Specific work }
\end{aligned}
$$

\section{Greek Symbols}

$$
\begin{aligned}
\beta & =\text { Panel angle } \\
\Gamma & =\text { Vortex strength } \\
\Delta S & =\text { Panel length } \\
\zeta_{\sigma} & =\text { Vorticity Gaussian distribution } \\
\theta & =\text { Clockwise angle starting from the stagnation point } \\
v & =\text { Kinematic viscosity } \\
\sigma & =\text { Core of a Lamb vortex } \\
\tau & =\text { Coordinate tangent to solid surface } \\
\chi & =\text { Random walk displacement } \\
\psi & =\text { Source strength per length } \\
\Omega & =\text { Fluid domain } \\
\omega & =\text { Vorticity field } \\
\omega & =\text { Component of the vorticity field }
\end{aligned}
$$

\section{Mathematical Formulation}

Consider the flow around a circular cylinder immersed in a large fluid region bounded by a moving plane surface, as shown in Fig. 1. A uniform incoming flow with freestream velocity $U$ from left to right is assumed. The fluid is Newtonian with constant properties and flowing in a two-dimensional plane; the compressibility effects are neglected. Figure 1 shows the domain $\Omega$ with boundary $S=S_{1} \cup S_{2} \cup S_{\infty}, S_{1}$ being the body surface, $S_{2}$ the moving plane running at the same speed as the incident flow and $\mathrm{S}_{\infty}$ the far away boundary.

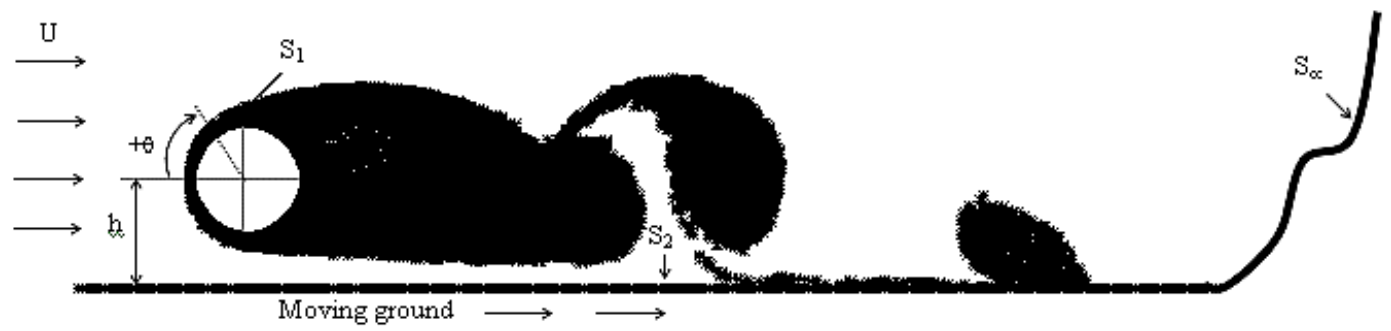

Figure 1. Flow around a circular cylinder near a moving ground.

Due to the no-slip condition, a shear flow is set on the cylinder surface and, as a consequence, vorticity is generated. The vorticity that develops in the body boundary layer is carried downstream into the viscous wake; further developments of this wake will be influenced by the presence of the nearby moving ground.

As there is no shear flow on the surface of the moving ground, no vorticity is generated as already mentioned. However, it is worth to mention the necessity of imposing the impermeability condition on this surface. The fluid flow is governed by the continuity and the Navier-Stokes equations, which can be written in the form: $\nabla \cdot \mathbf{u}=0$

$$
\frac{\partial \mathbf{u}}{\partial \mathrm{t}}+\mathbf{u} \cdot \nabla \mathbf{u}=-\nabla \mathrm{p}+\frac{1}{\operatorname{Re}} \nabla^{2} \mathbf{u}
$$

The above equations are non-dimensionalized in terms of $\mathrm{U}$ and $\mathrm{d}$ (cylinder diameter). The Reynolds number is defined by:

$$
\operatorname{Re}=\frac{\mathrm{Ud}}{\mathrm{v}}
$$


where $v$ is the fluid kinematic viscosity coefficient; the dimensionless time is $\mathrm{d} / \mathrm{U}$.

The impermeability condition on the cylinder and ground surfaces is given by:

$$
\mathrm{u}_{\mathrm{n}}=\mathrm{v}_{\mathrm{n}} \text {, at } \mathrm{S}_{1} \text { and } \mathrm{S}_{2} \text {. }
$$

The no-slip condition is imposed only on the cylinder surface:

$$
\mathrm{u}_{\tau}=\mathrm{v}_{\tau} \text {, at } \mathrm{S}_{1}
$$

In the equations above, $\mathrm{u}_{\mathrm{n}}$ and $\mathrm{u}_{\tau}$ are, respectively, the fluid normal and tangential velocities, and $\mathrm{v}_{\mathrm{n}}$ and $\mathrm{v}_{\tau}$ are, respectively, the solid boundary normal and tangential velocities. One assumes that, far away, the perturbation caused by the body and moving ground fades as:

$$
|\mathrm{u}| \rightarrow 1, \text { at } \mathrm{S}_{\infty}
$$

\section{The Numerical Method}

The governing equation in Vortex Methods is the vorticity transport equation, obtained by taking the curl of the momentum equation. In two-dimensions, this equation reduces to:

$$
\frac{\partial \omega}{\partial \mathrm{t}}+\mathbf{u} \cdot \nabla \omega=\frac{1}{\operatorname{Re}} \nabla^{2} \omega
$$

where $\omega(\mathbf{x}, \mathrm{t})=\nabla \times \mathbf{u}(\mathbf{x}, \mathrm{t})$ represents the only non-zero component of the vorticity field (observe that the pressure is absent from the formulation). The Vortex Method proceeds by discretizing spatially the vorticity field using a cloud of elemental vortices, which are characterized by a distribution of vorticity, $\zeta_{\sigma_{i}}$ (commonly called the cut-off function), the circulation strength $\Gamma_{\mathrm{i}}$ and the core size $\sigma_{\mathrm{i}}$. Thus, the discretized vorticity is expressed by:

$$
\omega(\mathbf{x}, \mathrm{t}) \approx \omega^{\mathrm{h}}(\mathbf{x}, \mathrm{t})=\sum_{\mathrm{i}=1}^{\mathrm{z}} \Gamma_{\mathrm{i}}(\mathrm{t}) \zeta_{\sigma_{\mathrm{i}}}\left(\mathbf{x}-\mathbf{x}_{\mathrm{i}}(\mathrm{t})\right)
$$

where $\mathrm{Z}$ is the number of point vortices of the cloud used to simulate the vorticity field.

In this paper, as the diffusion effects are simulated using the random displacement method, we assume that the core sizes are uniform $\left(\sigma_{i}=\sigma\right)$, and use the Gaussian distribution as the cut-off function. This choice of the cut-off function leads to the Lamb Vortices (Leonard, 1980); thus:

$$
\zeta_{\sigma}(\mathbf{x})=\frac{1}{\pi \sigma^{2}} \exp \left(-\frac{|\mathbf{x}|^{2}}{\sigma^{2}}\right)
$$

The velocity is obtained from the vorticity field by means of the Biot-Savart law:

$$
\begin{aligned}
& \operatorname{uv}(\mathbf{x}, \mathrm{t})=\int(\nabla \times \mathbf{G})\left(\mathbf{x}-\mathbf{x}^{\prime}\right) \omega\left(\mathbf{x}^{\prime}, \mathrm{t}\right) \mathrm{d} \mathbf{x}^{\prime}= \\
& =\int \mathbf{K}\left(\mathbf{x}-\mathbf{x}^{\prime}\right) \omega\left(\mathbf{x}^{\prime}, \mathrm{t}\right) \mathrm{d} \mathbf{x}^{\prime}=(\mathbf{K} * \omega)(\mathbf{x}, \mathrm{t})
\end{aligned}
$$

where $\mathbf{K}=\nabla \times \mathbf{G}$ is the Biot-Savart kernel, $\mathbf{G}$ is the Green's function for the Poisson equation, and $*$ represents the convolution operation.
The vorticity transport is simulated, in this discretized form, by convecting the particles with the local fluid velocity and using a random walk displacement $\chi_{\mathrm{j}} \equiv\left(\chi_{1 \mathrm{j}}, \chi_{2 \mathrm{j}}\right)$ to account for the diffusion effects.

The convection of each vortex particle $(j)$ is governed by the equation:

$$
\frac{\mathrm{d} \mathbf{x}_{\mathrm{j}}}{\mathrm{dt}}=\mathbf{u}\left(\mathbf{x}_{\mathrm{j}}, \mathrm{t}\right)
$$

and, according to the Random Walk Method (Lewis, 1999), the diffusive displacement of each vortex particle (j) is given by:

$$
\chi_{\mathrm{j}} \equiv\left(\chi_{1 \mathrm{j}}, \chi_{2 \mathrm{j}}\right)=\sqrt{\frac{4 \Delta \mathrm{t}}{\mathrm{Re}} \ln \left(\frac{1}{\mathrm{P}}\right)}[\cos (2 \pi \mathrm{Q})+\mathrm{i} \sin (2 \pi \mathrm{Q})]
$$

where $\mathrm{j}=1, \mathrm{Z}, \mathrm{i}^{2}=-1, \mathrm{P}$ and $\mathrm{Q}$ are random numbers between 0.0 and 1.0.

The velocity field $\mathbf{u}(\mathbf{x}, \mathbf{t})$ can be split in three parts (Alcântara Pereira et al., 2003):

$$
\mathbf{u}(\mathbf{x}, \mathrm{t})=\mathbf{u i}(\mathbf{x}, \mathrm{t})+\mathbf{u b}(\mathbf{x}, \mathrm{t})+\mathbf{u v}(\mathbf{x}, \mathrm{t}) .
$$

The contribution of the incident flow is represented by ui $(\mathbf{x}, \mathbf{t})$. For a uniform incoming flow its components take the form:

$$
\mathrm{ui}_{1}=1 \text { and } \mathrm{ui}_{2}=0 \text {. }
$$

The body and moving ground contribute with $\mathbf{u b}(\mathbf{x}, \mathrm{t})$, which can be obtained, for example, using the Boundary Element Method (Katz and Plotkin, 1991). The two components can be written as:

$$
\mathrm{ub}_{\mathrm{i}}\left(\mathrm{x}_{\mathrm{j}}, \mathrm{t}\right)=\sum_{\mathrm{k}=1}^{\mathrm{NP}} \psi_{\mathrm{k}} \mathrm{c}_{\mathrm{jk}}^{\mathrm{i}}\left(\mathrm{x}_{\mathrm{j}}(\mathrm{t})-\mathrm{x}_{\mathrm{k}}\right), \mathrm{i}=1,2 \text { and } \mathrm{j}=1, \mathrm{Z}
$$

where NP is the total number of flat source panels representing the body and moving ground. It is assumed that the source strength per length is constant such that $\psi_{k}=$ const and $c_{j k}^{i}\left(x_{j}(t)-x_{k}\right)$ is the $i^{\text {th }}$ component of the velocity induced, at vortex $\mathrm{j}$, by a unit strength flat source panel located at $\mathrm{k}$.

Finally, the velocity $\mathbf{u v}(\mathbf{x}, \mathbf{t})$ due to the vortex interactions has its components written as:

$$
u v_{i}\left(x_{j}, t\right)=\sum_{k=1}^{Z} \Gamma_{k} c_{j k}^{i}\left(x_{j}(t)-x_{k}(t)\right), i=1,2 \text { and } j=1, Z \text {. }
$$

where $\Gamma_{k}$ is the k-vortex strength and $c_{j k}^{i}\left(x_{j}(t)-x_{k}\right)$ is the $i^{\text {th }}$ component of the velocity induced, at vortex $\mathrm{j}$, by a unit strength vortex located at $\mathrm{k}$. As we use the Lamb vortex:

$$
\mathrm{u}_{\theta}^{\mathrm{jk}}=-\frac{\Gamma_{\mathrm{k}}}{2 \pi} \frac{1}{\mathrm{r}_{\mathrm{jk}}}\left[1-\exp \left(-5.02572 \frac{\mathrm{r}_{\mathrm{jk}}^{2}}{\sigma_{0}^{2}}\right)\right]
$$

where $\mathrm{u}_{\theta}^{\mathrm{jk}}$ is the induced velocity of the $\mathrm{k}^{\text {th }}$-vortex in the circumferential direction at $\mathrm{j}^{\text {th }}$-vortex, $r_{\mathrm{jk}}$ is the radial distance between two general vortices, $\mathrm{j}$ and $\mathrm{k}$, and $\sigma_{0}$ is the core radius of these vortices defined by Mustto et al.(1998).

To the first order Euler scheme, the solution to Eq. (11) is written as: 


$$
\begin{aligned}
& x_{j}(t+\Delta t)=x_{j}(t)+u_{j}(x, t) \Delta t, j=1, Z \\
& y_{j}(t+\Delta t)=y_{j}(t)+v_{j}(x, t) \Delta t, j=1, Z
\end{aligned}
$$

To this solution, the diffusive displacement, see Eq. (12), is added. Hence, the position of each vortex at the instant $(t+\Delta t)$ is given by:

$$
\begin{aligned}
& x_{j}(t+\Delta t)=x_{j}(t)+u_{j}(x, t) \Delta t+\chi_{1 j}, j=1, Z \\
& y_{j}(t+\Delta t)=y_{j}(t)+v_{j}(x, t) \Delta t+\chi_{2 j}, j=1, Z .1
\end{aligned}
$$

With the vorticity field, the pressure calculation starts with the Bernoulli function, defined by Uhlman (1992) as:

$$
\overline{\mathrm{Y}}=\mathrm{p}+\frac{\mathrm{u}^{2}}{2}, \mathrm{u}=|\mathbf{u}|
$$

Kamemoto (1993) used the same function, and starting from the Navier-Stokes equations was able to write a Poisson equation for the pressure. This equation was solved using a finite difference scheme. Here solution was obtained through the following integral formulation (Shintani and Akamatsu, 1994):

$$
\begin{aligned}
& H \bar{Y}_{i}-\int_{S} \bar{Y} \nabla G_{i} \cdot \mathbf{e}_{n} d S=\iint_{\Omega} \nabla G_{i} \cdot(\mathbf{u} \times \boldsymbol{\omega}) \mathrm{d} \Omega+ \\
& -\frac{1}{\operatorname{Re}} \int_{S}\left(\nabla G_{i} \times \boldsymbol{\omega}\right) \cdot \mathbf{e}_{\mathrm{n}} \mathrm{dS}
\end{aligned}
$$

where $\mathrm{H}=1.0$ in the fluid domain, $\mathrm{H}=0.5$ on the boundaries, $\mathrm{G}$ is a fundamental solution of the Laplace equation and $\mathbf{e}_{\mathrm{n}}$ is the unit vector normal to the solid surfaces (Alcântara Pereira et al., 2002).

The drag and lift coefficients can be expressed by (Ricci, 2002):

$$
\begin{aligned}
& \mathrm{C}_{\mathrm{D}}=-\sum_{\mathrm{k}=1}^{\mathrm{NP}} 2\left(\mathrm{p}_{\mathrm{k}}-\mathrm{p}_{\infty}\right) \Delta \mathrm{S}_{\mathrm{k}} \sin \beta_{\mathrm{k}}= \\
& -\sum_{\mathrm{k}=1}^{\mathrm{NP}} \mathrm{C}_{\mathrm{P}} \Delta \mathrm{S}_{\mathrm{k}} \sin \beta_{\mathrm{k}} \\
& \mathrm{C}_{\mathrm{L}}=-\sum_{\mathrm{k}=1}^{\mathrm{NP}} 2\left(\mathrm{p}_{\mathrm{k}}-\mathrm{p}_{\infty}\right) \Delta \mathrm{S}_{\mathrm{k}} \cos \beta_{\mathrm{k}}= \\
& -\sum_{\mathrm{k}=1}^{\mathrm{NP}} \mathrm{C}_{\mathrm{P}} \Delta \mathrm{S}_{\mathrm{k}} \cos \beta_{\mathrm{k}}
\end{aligned}
$$

where $\Delta \mathrm{S}_{\mathrm{k}}$ is the length and $\beta_{\mathrm{k}}$ is the angle and both of the $\mathrm{k}^{\text {th }}$-panel.

\section{Simulations of Unsteady Flows past a Circular Cylinder}

\section{Isolated Cylinder}

As a preliminary study, the flow around an isolated cylinder in a large fluid region was simulated using our numerical code. This allows us to analyze its consistency and define some numerical parameters, as for example the number of panels used to define the cylinder surface. For this particular configuration, we used NP $=300$ flat source panels with constant density. The simulation was performed up to 1000 time steps with magnitude $\Delta t=0.05$. The time increment was evaluated according to $\Delta \mathrm{t}=2 \pi \mathrm{k} / \mathrm{NP}, 0<\mathrm{k}<1$; see Mustto et al. (1998).

The standard numerical strategy is to represent the vorticity in the fluid domain by a large number $\mathrm{Z}$ of discrete vortices with strength $\Gamma_{\mathrm{j}}$. The numerical analysis is conducted over a series of discrete time steps $\Delta \mathrm{t}$ for each of which a discrete vortex element with strength $\Gamma_{\mathrm{j}}$ is shed from each panel used to represent the cylinder surface. The intensity $\Gamma_{j}$ of these newly generated vortices is determined using the no-slip condition, see Eq. (5), and they are placed at a distance $\varepsilon=\sigma_{0}=0.001 \mathrm{~d}$ on a straight-line normal to the panel, see Ricci (2002).

The aerodynamic loads computations are evaluated between $\mathrm{t}=$ 28.3 and $t=48.0$, see Fig. 2. The results of the numerical simulation are presented in Tab. 1; the results of Blevins (1984) are experimental ones with $10 \%$ uncertainty and those of Mustto et al. (1998) were obtained numerically using a slightly different Vortex Method from the present implementation.

Table 1. Mean lift and drag coefficients for isolated circular cylinder.

\begin{tabular}{|c|c|c|c|c|}
\hline $\operatorname{Re}=1.0 \times 10^{5}$ & $\overline{\mathrm{C}_{\mathrm{D}}}$ & $\overline{\mathrm{C}_{\mathrm{L}}}$ & $\overline{\mathrm{St}}$ & $\overline{\mathrm{A}}_{\mathrm{C}_{\mathrm{L}}}$ \\
\hline Blevins (1984) & 1.20 & - & 0.19 & - \\
\hline Mustto et al. (1998) & 1.22 & - & 0.22 & - \\
\hline Present Simulation & 1.25 & 0.02 & 0.21 & 1.06 \\
\hline
\end{tabular}

The Strouhal number is defined as:

$$
S_{t}=\frac{f d}{U}
$$

where $\mathrm{f}$ is the detachment frequency of vortices.

The agreement between the two numerical methods is very good for the Strouhal number, and both results are close to the experimental value. The present drag coefficient shows a higher value as compared to the experimental result. One should observe that the three-dimensional effects are non-negligible for the Reynolds number used in the present simulation $\left(\operatorname{Re}=1.0 \times 10^{5}\right)$. Therefore, one can expect that a two-dimensional computation of such a flow must produce higher values for the drag coefficient. On the other hand, the Strouhal number is insensitive to these threedimensional effects. The mean numerical lift coefficient, although very small, is not zero which is due to numerical approximations.

Computed values for the drag and lift coefficients are plotted in Fig. 2. The vortex shedding effect can be seen in the oscillations of the lift and drag coefficients. As soon as the numerical transient is over and the periodic regime is reached (from $\mathrm{t}=20 \mathrm{on}$, approximately) the lift coefficient oscillates between -1.11 and 1.01, approximately, with a dimensionless frequency (Strouhal number) that is one half the frequency of oscillation of the drag coefficient curve, as expected. The mean amplitude of the lift coefficient curve is indicated by $\overline{\mathrm{A}_{\mathrm{C}_{\mathrm{L}}}}$ in Tab. 1. 


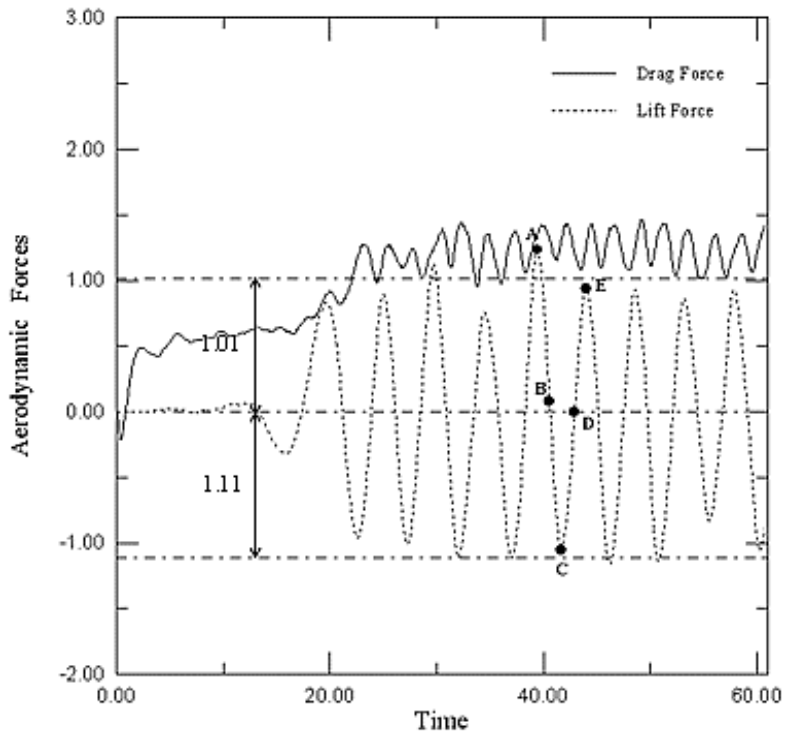

Figure 2. Time history of drag and lift coefficients for isolated circular cylinder.

Figure 3 shows plots of instantaneous pressure distributions on the cylinder surface. Distributions A, B, C, D and E are related to instants A, B, C, D and E as indicated in Fig. 2.

Instant $\mathrm{A}$ is defined by a maximum value of the lift coefficient. At this moment, a large clockwise vortex structure (in fact a cluster of vortices) is detaching from the upper surface and moving towards the viscous wake; see Fig. 4(a). As this clockwise vortex structure moves downstream, it pushes away an anti-clockwise structure that was stationed behind the cylinder, and the drag coefficient increases.

Instant $\mathrm{B}$ is defined as the moment that the anti-clockwise structure detaches from the cylinder and is incorporated into the viscous wake; this process creates a low pressure region at the rear part of the cylinder; see Fig. 3 and Fig. 4(b).

The above described sequence of events repeats all over again. Therefore, the lowest value of the lift coefficient is observed when another cluster, now rotating in the anti-clockwise direction, leaves the body surface, see point C in Fig. 3 and Fig.4(c), and point D in Fig 4(d).

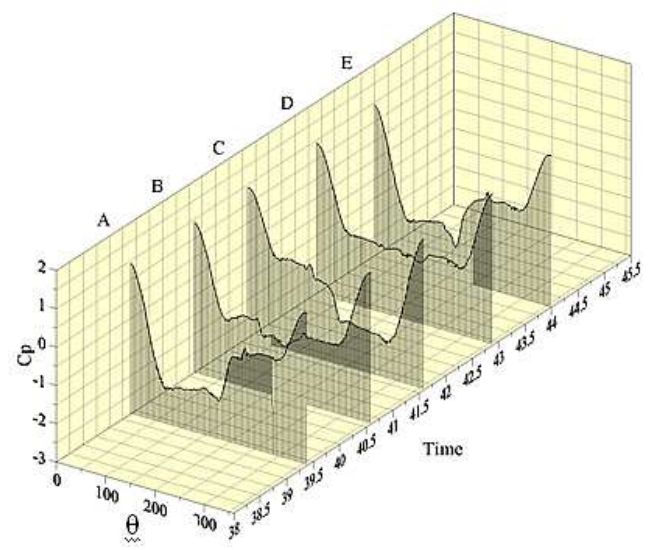

Figure 3. Instantaneous pressure distribution on the surface of an isolated circular cylinder.
Gerrard (1966) has given an equivalent physical description of the mechanics of the vortex-formation region. A key factor in the formation of a vortex-street wake is the mutual interaction between the two separating shear layers. It is postulated by Gerrard (1966) that a vortex continues to grow fed by circulation from its connected shear layers, until it is strong enough to draw the opposing shear layers across the near wake. The approach of oppositely signed vorticity, in sufficient concentration, cuts off further supply of circulation to the growing vortex, which is then shed and moves off downstream.

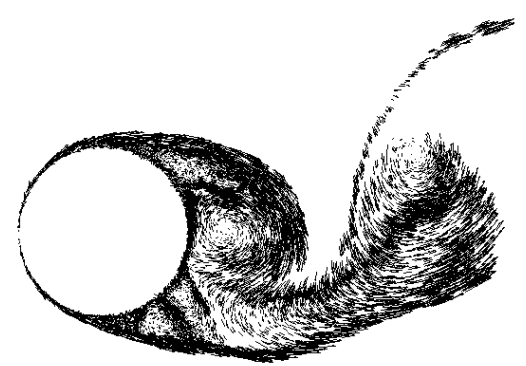

(a) $\mathrm{t}=39.4$ : Point $\mathrm{A}$

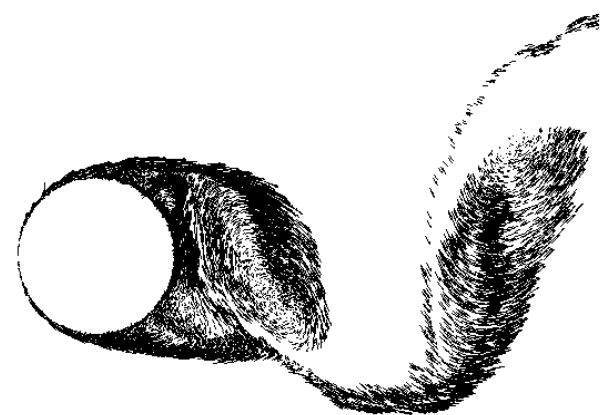

(b) $\mathrm{t}=40.6$ : Point $\mathrm{B}$

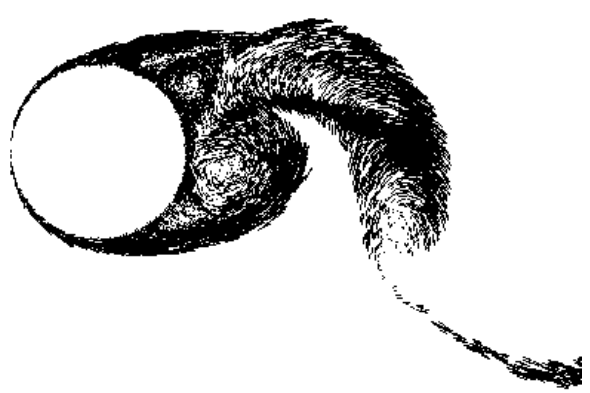

(c) $\mathrm{t}=41.6$ : Point $\mathrm{C}$

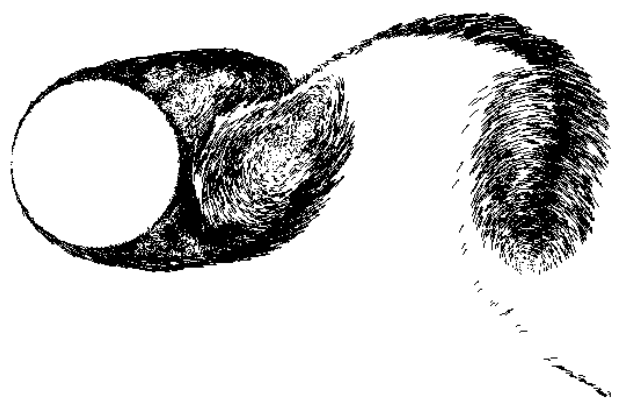

(d) $\mathrm{t}=$ 42.9: Point D

Figure 4. Near wake behavior for isolated cylinder at $R e=1.0 \times 10^{5}$. 
Computed value of the mean pressure coefficient along the cylinder surface is compared with other results available in the literature. Figure 5 shows the mean pressure distribution calculated for an isolated cylinder to be compared with the potential flow pressure distribution, the pressure distribution presented by Mustto et al. (1998) and the experimental values presented by Blevins (1984). The present result agrees very well with the experimental ones, except in a small neighborhood of $\theta \sim 75^{\circ}$. From the Fig. 5, one can observe that the predicted separation point occurs at around $\theta=85^{\circ}$, while the experimental value (Blevins, 1984) is around $\theta=82^{\circ}$. Another experimental investigation made by Son and Hanratty (1969) determined a value of $\theta=78^{\circ}$ for the separation angle. A very interesting observation was made by Achenbach (1968) for $\mathrm{Re}=1.0 \times 10^{5}$ (sub-critical flow): it was found that the laminar boundary layer separates at $\theta=78^{\circ}$. Just before transition into the critical region at $\operatorname{Re}=2.6 \times 10^{5}$, the boundary layer is still laminar and separates at an angle equal $\theta=$ $94^{\circ}$. Hence, separation takes place in the laminar mode as experimentally expected for a sub-critical Reynolds number forming free shear layer. An immediate transition to turbulence close to the cylinder is observed accompanied by a very short recirculation region.

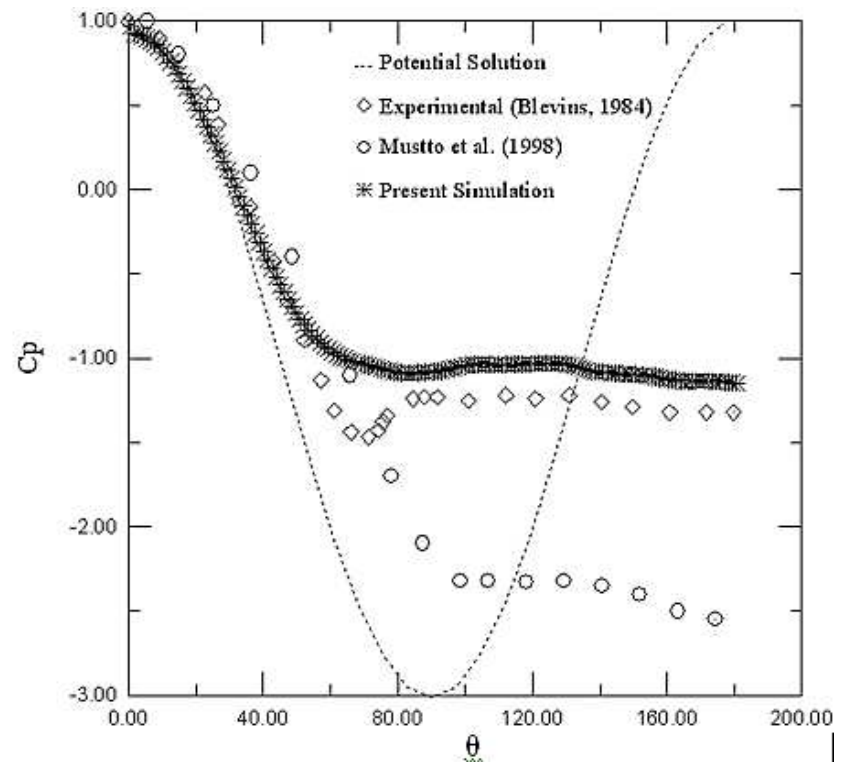

Figure 5. Predicted pressure distributions for isolated circular cylinder at $\mathrm{Re}=1.0 \times 10^{5}$.

\section{Circular Cylinder near a Moving Ground}

To study the mechanisms of the ground effect, we use a ground running at the speed of the freestream flow. In doing so, no boundary layer develops on the ground surface to interfere with and to modify the viscous wake. The main features of this flow are discussed in the experimental work of Nishino (2007). Although the fundamental effects of the gap ratio (h/d) on the flow and force characteristics have been observed, the relation between the destruction of the orderly Kármán vortex street and the significant drag reduction is still unclear.

For the numerical simulation we used the same 300 panels for the cylinder surface plus 300 panels to represent the moving ground. As already mentioned, no vorticity is generated on the ground surface which avoids the development of a viscous boundary layer.
Table 2 presents values of the drag coefficient for a circular cylinder placed at different values of the gap ratio. One can easily observe three gap regimes: large-gap $(\mathrm{h} / \mathrm{d}>1.0)$, intermediate-gap $(0.85<\mathrm{h} / \mathrm{d}<1.0)$, and small-gap $(\mathrm{h} / \mathrm{d}<0.85)$ regimes.

Nishino (2007) measured the drag coefficient at two uppersubcritical Reynolds numbers $\left(0.4\right.$ and $\left.1.0 \times 10^{5}\right)$. According to him, an essentially two-dimensional flow around a cylinder with endplates was observed, which was confirmed analyzing the surface oil flow patterns. Significant effects of the gap ratio were observed on the near wake structure and also on the time-averaged drag coefficient. For the large-gap regime, large-scale Kármán vortices were generated just behind the cylinder, resulting in higher drag coefficients of about 1.3. For the intermediate-gap regime, the Kármán vortex shedding became intermittent, and hence the timeaveraged drag coefficient rapidly decreased as h/d was reduced from 1.0 to 0.85 . For the small-gap regime, the Kármán vortex could not be observed and instead a dead fluid zone was created, bounded by two nearly parallel shear layers each producing only small-scale vortices. For the cylinder without end-plates, on the other hand, no such significant effects of $\mathrm{h} / \mathrm{d}$ were observed either on the near wake structure or on the drag coefficient.

Roshko et al. (1975) measured the time-averaged drag and lift coefficients for a circular cylinder placed near a fixed wall in a wind tunnel at $\operatorname{Re}=2.0 \times 10^{4}$, which lies in the upper-subcritical flow regime, and showed that $C_{D}$ decreases rapidly while $C_{L}$ increases as the cylinder came close to the wall.

Columns 5 and 6 of Tab. 2 present results obtained using numerical methods. The results of Moura (2007) were obtained using the vortex cloud simulation with fixed ground at $\operatorname{Re}=1.0 \times 10^{5}$. The present results, referred to as Bimbato (2008), for the time-averaged drag and lift coefficients acting on a circular cylinder in moving ground are plotted in Fig. 6 . The aerodynamic forces computations are evaluated between $\mathrm{t}=40$ and $\mathrm{t}=60$.

The following analysis for the drag behavior is based on Fig. 6.a.

The results from Nishino (2007), obtained with a running ground, show that the drag acting on the cylinder without end-plates increases and becomes more or less constant when the distance between the cylinder and the ground is very small. A cylinder with end-plates presents an almost constant value for the drag coefficient, but higher than the case when the end-plates are not used; it is worth to observe that in this situation, due to experimental difficulties, he was not able to perform the tests for small-gap regime.

The results presented by Roshko et al. (1975) show that drag decreases as the gap-ratio decreases, starting already for the intermediate-gap regime; these results were obtained with a fixed ground.

The numerical results for the drag obtained with a fixed ground by Moura (2007) follows the experimental ones for the large and intermediate-gap regimes but does not reproduce them well for the small-gap regime.

The present results, obtained with a running ground, show that the drag remains an almost constant value for the large and intermediate-gap regimes as predicted by the experiments of Nishino (2007); the values are a little higher, however. For the small-gap regime, the drag decreases as the gap-ratio decreases and, unfortunately, there are no experimental results to compare with. It is interesting to observe that the drag coefficient converges to the same value obtained experimentally by Nishino (2007), without end-plates, for very small gap-ratio. 
Table 2. Summary of results for drag coefficient on the flow around a circular cylinder near a plane boundary.

\begin{tabular}{c|c|c|c|c|c}
$\mathrm{h} / \mathrm{d}$ & $\begin{array}{c}\text { Nishino (2007) } \\
\text { without end-plates }\end{array}$ & $\begin{array}{c}\text { Nishino (2007) } \\
\text { with end-plates }\end{array}$ & $\begin{array}{c}\text { Roshko et al. } \\
(1975)\end{array}$ & $\begin{array}{c}\text { Moura } \\
(2007)\end{array}$ & $\begin{array}{c}\text { Bimbato } \\
(2008)\end{array}$ \\
\hline 0.50 & - & - & 0.795 & - & - \\
\hline 0.55 & 0.965 & - & 0.857 & 1.809 & 1.154 \\
\hline 0.60 & 0.958 & - & - & - & 0.832 \\
\hline 0.65 & 0.952 & - & 0.954 & 1.656 & 1.293 \\
\hline 0.70 & 0.939 & - & - & - & 1.376 \\
\hline 0.75 & 0.933 & - & 1.029 & 1.440 & 1.406 \\
\hline 0.80 & 0.930 & - & - & - & 1.393 \\
\hline 0.85 & 0.931 & - & - & - & 1.415 \\
\hline 0.90 & 0.922 & - & 1.136 & 1.365 & 1.421 \\
\hline 0.95 & 0.926 & 1.311 & - & 1.453 & 1.403 \\
\hline 1.00 & 0.924 & 1.323 & - & 1.491 & 1.391 \\
\hline 1.10 & 0.920 & 1.373 & 1.281 & 1.466 & 1.383 \\
\hline 1.30 & 0.899 & 1.385 & - & 1.410 & 1.362 \\
\hline 1.40 & - & - & 1.266 & - & - \\
\hline 1.50 & 0.881 & 1.375 & - & 1.385 & 1.346 \\
\hline 2.00 & 0.854 & 1.337 & - & 1.346 & 1.277 \\
\hline 2.30 & - & - & 1.243 & - & - \\
\hline 2.50 & 0.845 & 1.304 & - & - & 1.269 \\
\hline 3.50 & - & - & 1.234 & - & -
\end{tabular}

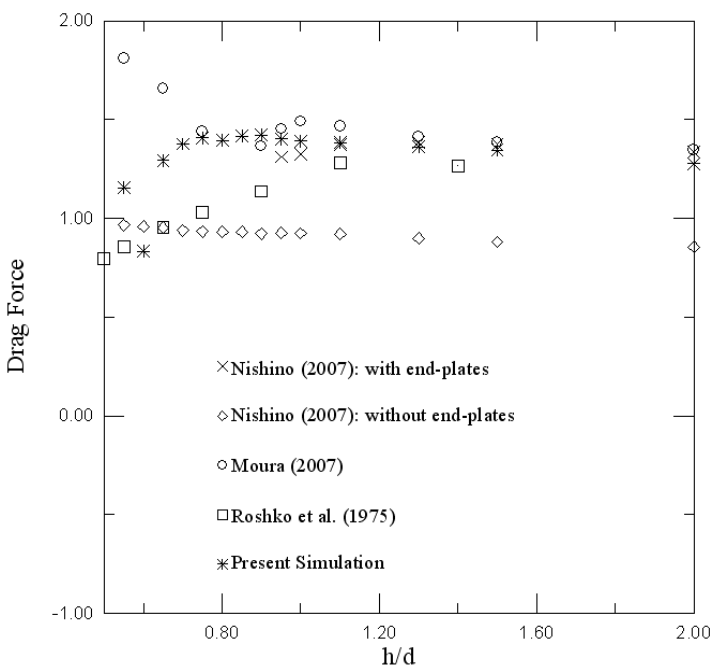

(a) Drag force

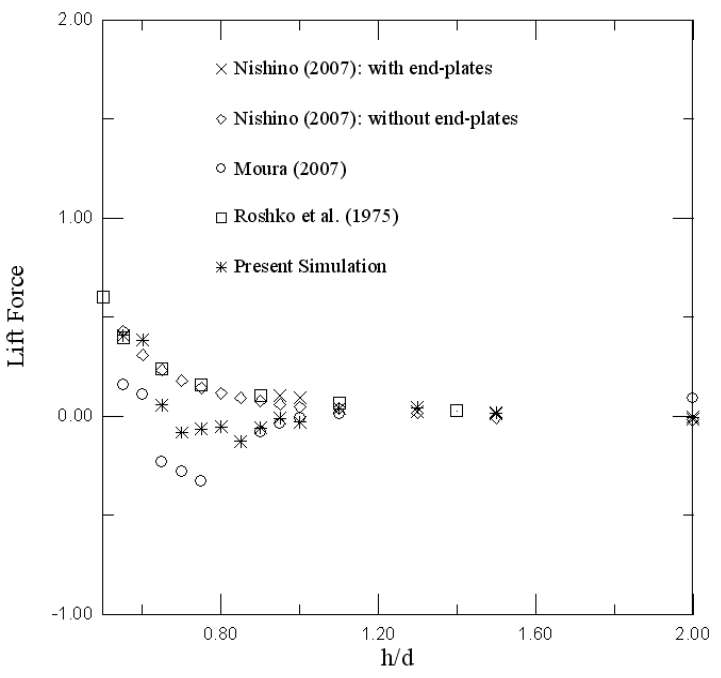

(b) Lift force

Figure 6. Time-averaged drag and lift coefficients vs. gap ratio for different end conditions.
Figure 6(b) shows that the lift coefficient curve obtained numerically follows quite well the values obtained experimentally, except when $0.7<\mathrm{h} / \mathrm{d}<1.0$, where the calculated values are smaller. For smaller values of the gap-ratio, there are no experimental values available when the end-plates are added to the cylinder. However, it is worth to observe that all the experimental and numerical results indicate the same limiting value for really small gap-ratio.

Figure 7 shows the instantaneous pressure distributions on the cylinder surface when the ground is moving; this sample refers to the gap-ratio $\mathrm{h} / \mathrm{d}=0.95$. The pressure distributions $\mathrm{A}, \mathrm{B}, \mathrm{C}, \mathrm{D}$ and $\mathrm{E}$ are related to points $\mathrm{A}, \mathrm{B}, \mathrm{C}, \mathrm{D}$ and $\mathrm{E}$ indicated in Fig. 8. At the instant represented by the point $\mathrm{A}$ one can observe a low pressure distribution on the rear surface of the cylinder, leading to a maximum value of the drag curve; at the same time, a high pressure distribution is found on the lower surface which leads to a high lift value. The pressure distribution of instant $B$ is almost symmetrical with respect to the $\mathrm{x}$ axis while maintaining low values at the rear part, thus explaining the zero value of the lift curve. Similar observations can be made about the pressure distributions and the lift and drag curves behavior at the other instants.

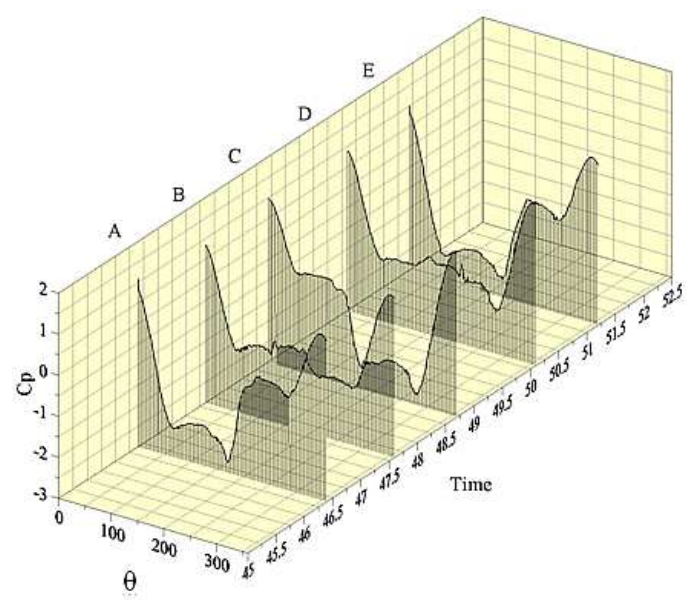

Figure 7. Instantaneous pressure distribution on the surface of a circular cylinder using moving ground for $\mathrm{h} / \mathrm{d}=\mathbf{0 . 9 5}$. 
Some important features of the curves presented in Fig. 8 are:

(i) As expected, the absolute value of the maximum of the $\mathrm{C}_{\mathrm{L}}$ curve is bigger than the absolute value of the minimum of the same curve.

(ii) The $C_{D}$ curve oscillates at a frequency that is twice the frequency of the $C_{L}$ curve.

(iii) Due to the proximity of the moving ground, the $C_{D}$ curve, in Fig. 8, presents a pair of small extreme values (small departure of the maximum and minimum values from the mean drag value) followed by a pair of large extreme values (large departure of the maximum and minimum values from the mean drag value).

(iv) As the gap-ratio diminishes, the small extreme values become even smaller and eventually disappear. Therefore, the drag and lift curves oscillate at the same frequency.

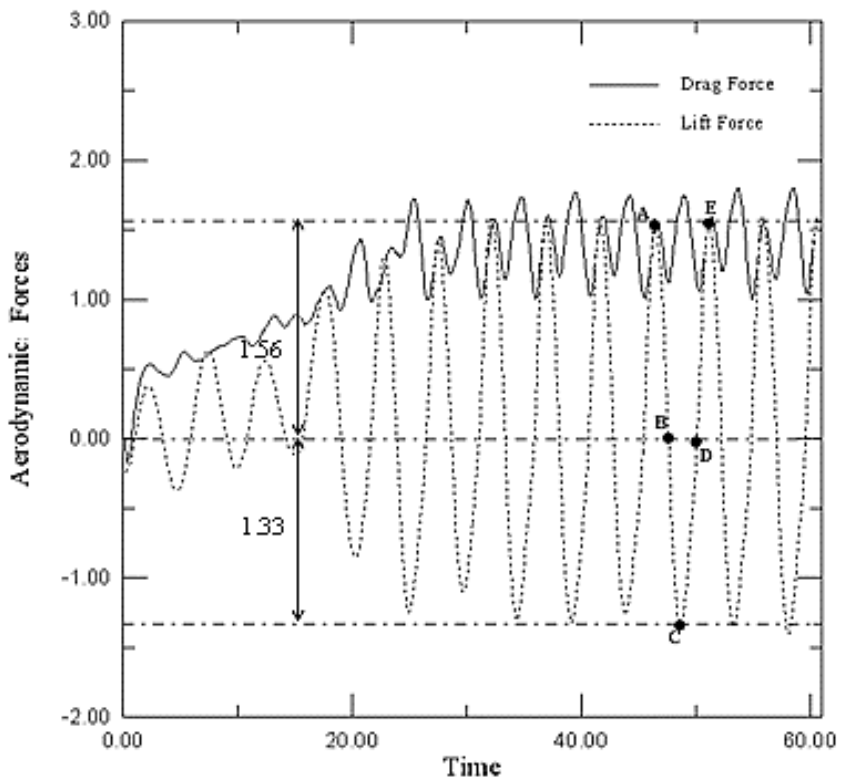

Figure 8. Time history of drag and lift for circular cylinder using moving ground for $h / d=0.95$.

Figure 9(a) shows the near field flow pattern at instant A; at this instant, we observe a maximum value of the $C_{L}$ curve and a "small" maximum value of the $C_{D}$ curve. The analysis of the flow pattern at instants right before and after the instant $\mathrm{A}$ shows that a cluster of vortices is moving on the upper side of the cylinder surface (leading to a high value of the lift) and pulls the anti-clockwise vortex structure toward the viscous wake. This vortex structure is deformed and somehow stretched by the presence of the nearby moving ground, leading to a "small" maximum value of the drag curve.

A similar analysis can be done for all the other instants identified in Fig. 8; the near field flow pattern for those instants are shown in Fig. 9. For instance, in Fig. 9(b), the near field flow pattern at instant B is depicted. At this instant, a clockwise vortex structure is observed at the rear part of the cylinder surface; this clockwise structure is deformed when it pulls the anti-clockwise structure away from the body surface. This configuration is the one responsible for a "small" minimum value of the $\mathrm{C}_{\mathrm{D}}$ curve and a zero value of the $C_{L}$ curve. Figures $9(c)$ and $9(d)$ are associated to instants $C$ and $D$ at which the extreme values of the $C_{D}$ are "large"; observe that the near field vortex structures do not deform.
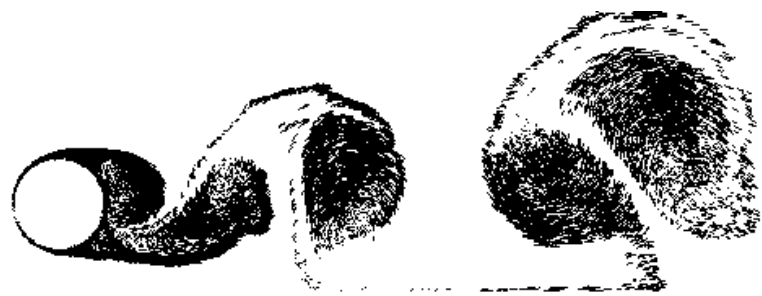

(a) $\mathrm{t}=46.4$ : Point $\mathrm{A}$
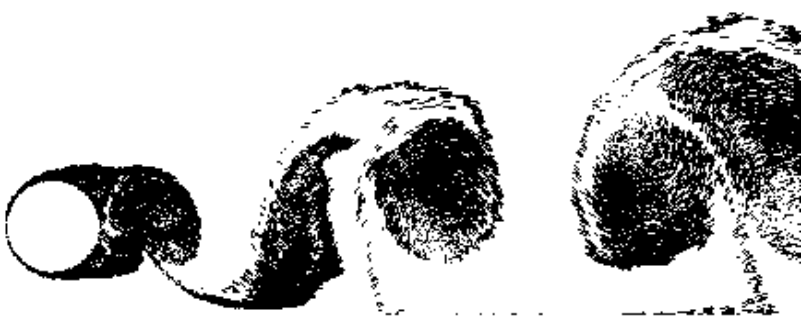

(b) $t=47.6$ : Point B
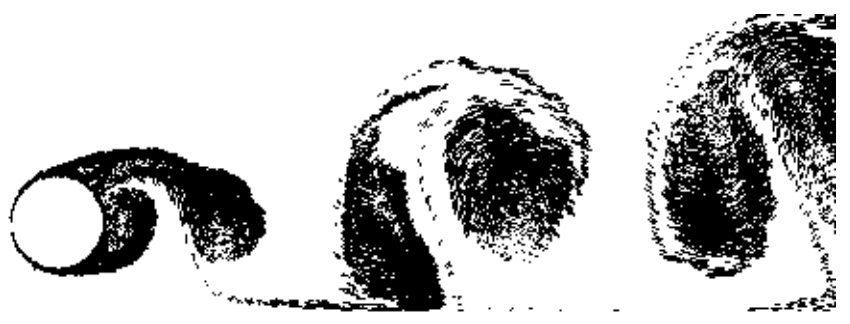

(c) $\mathrm{t}=48.7$ : Point $\mathrm{C}$

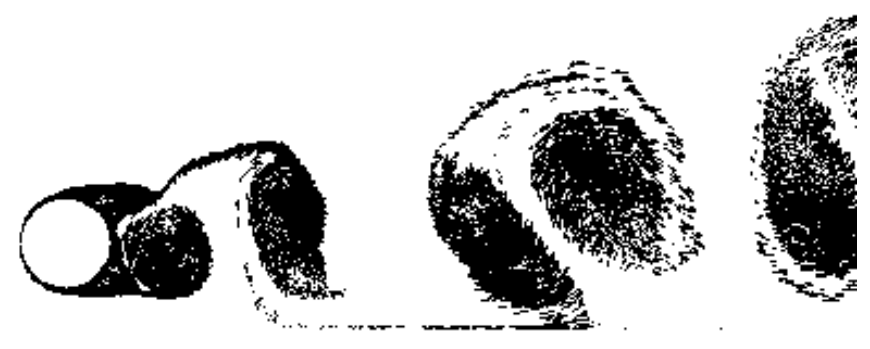

(d) $t=50.1$ : Point D

Figure 9. Near wake behavior using moving ground for $h / d=0.95$ at $R e=1.0 \times 10^{5}$.

Figure 10 shows the time variation of the drag and lift coefficients for $h / d=0.55$. From this figure one can observe the tendency to the cessation of the periodic vortex shedding due to the presence of a plane wall placed in the close vicinity of the cylinder. After all, the gap between the lowest point of the cylinder and the wall is equal to $0.05 \mathrm{~d}$. 


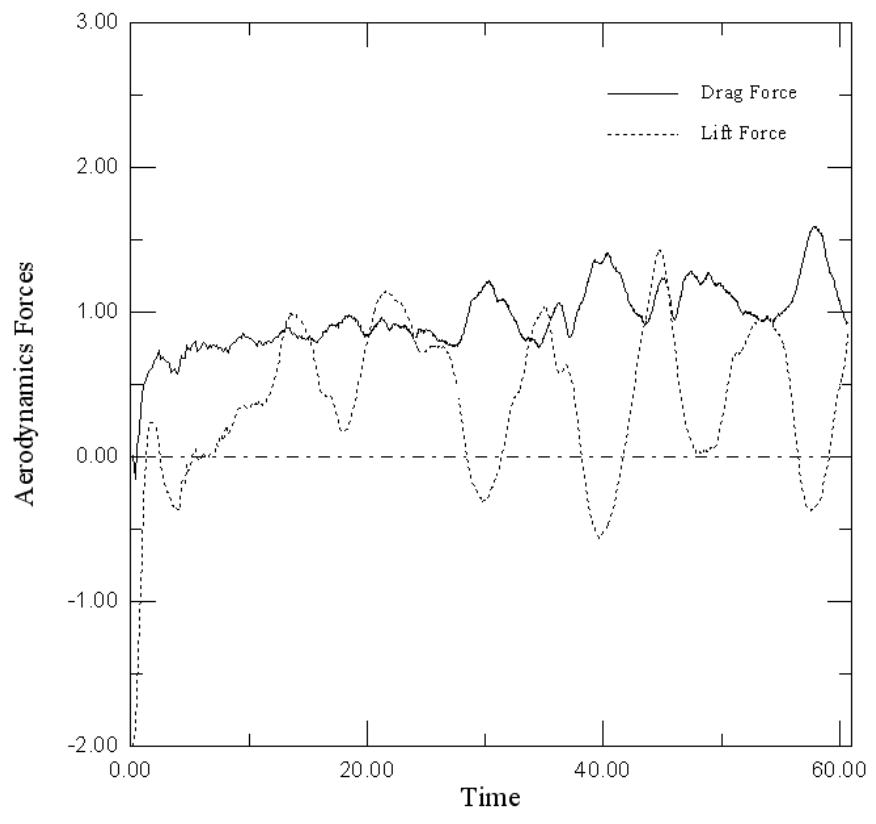

Figure 10. Time history of drag and lift for a circular cylinder using moving ground for $h / d=0.55$.

Just for the sake of illustration, the flow pattern at instant $t=62$ is shown in Fig. 11 for two gap-ratios $[(\mathrm{h} / \mathrm{d})=0.55$ and $(\mathrm{h} / \mathrm{d})=$ 0.95]. For really small gap, Fig. 11(a), the vortex shedding becomes intermittent, which might be an explanation for the fast decay of the time-averaged drag coefficient as observed in the experiments from Nishino (2007). For a not so small gap, Fig. 11(b), the wake seems to be formed by a series of "mushroom" type of vortex structure, which will be destroyed far away by the moving ground.

\section{Conclusions}

The main conclusions that can be drawn are:

(i) As already used in the experimental work dealing with the aerodynamic of high speed racing cars, the moving ground model used in the numerical simulations (although with a simple geometrical form body) is able to predict the main features of the flow around a body in close proximity of a flat surface.

(ii) The experience gained with the present work added to the ones from previous one, in which the ground was kept fixed, allows one to analyze complex situations, where relative motions between bodies are present. These extend the applicability of the numerical code.

(iii) The use of global as well as local quantities combined to the near field flow pattern observations can be used to understand the complex mechanisms that lead the origin and the time evolution of the aerodynamic loads. The methodology developed in this paper is greatly simplified by the utilization of the Vortex Method.

(iv) The instantaneous pressure distribution on the cylinder surface allows one to follow, in time, its evolution. This feature can be of importance when the body is oscillating near a ground plane and in many other situations of practical interest. It becomes obvious that one has a powerful tool if the time evolution of the pressure distribution is analyzed simultaneously with the integrated loads (lift and drag).

(v) Further analyses are necessary to fully understand the drag behavior as well as the wake development when the body is brought close to a ground. Fig. 11 gives us only some hints.

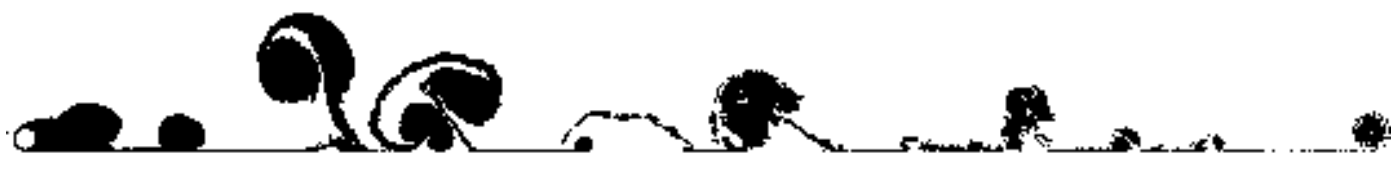

(a) $\mathrm{h} / \mathrm{d}=0.55$

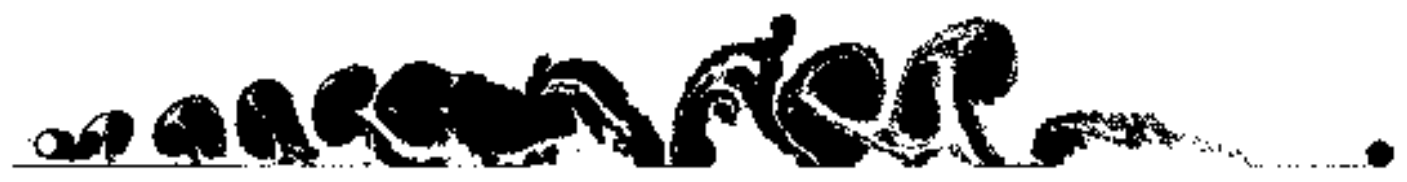

(b) $h / d=0.95$

Figure 11. Final position of the vortices for the flow past a circular cylinder in moving ground at $R e=1.0 \times 10^{5}$.

\section{Acknowledgements}

This research was supported by the CNPq (Brazilian Research Agency) Proc. 470420/2008-1, FAPERJ (Research Foundation of the State of Rio de Janeiro) Proc. E-26/112/013/2008 and FAPEMIG (Research Foundation of the State of Minas Gerais) Proc. TEC APQ-01074-08.

\section{References}

Achenbach, E., 1968, "Distribution of Local Pressure and Skin Friction around a Circular Cylinder in Cross-Flow up to", J. Fluid Mech., Vol. 34, No. 4, pp. 625-639.

Alcântara Pereira, L.A., Ricci, J.E.R., Hirata, M.H., and Siveira Neto, A., 2002, "Simulation of Vortex-Shedding Flow around a Circular Cylinder 
with Turbulence Modelling", International Society of CFD/CFD Journal, Vol. 11, No. 3, pp. 315-322.

Alcântara Pereira, L.A., Hirata, M.H., and Silveira Neto, A., 2003, "Vortex Method with Turbulence Sub-Grid Scale Modeling", J. of the Braz. Soc. of Mech. Sci. \& Eng. Vol. 25, No. 1, pp. 140-146.

Alcântara Pereira, L.A., Hirata, M.H., and Manzanares Filho, N., 2004,

"Wake and Aerodynamics Loads in Multiple Bodies - Application to Turbo Machinery Blade Rows", J. of Wind Engineering and Industrial Aerodynamics, Vol. 92, pp. 477-491.

Angrilli, F., Bergamaschi, S., and Cossalter, V., 1982, "Investigation of Wall Induced Modifications to Vortex Shedding from a Circular Cylinder", Transactions of the ASME: Journal of Fluids Engineering, Vol. 104, pp. 518-522.

Bearman, P.W., and Zdravkovich, M.M., 1978, "Flow around a Circular Cylinder near a Plane Boundary", Journal of Fluid Mechanics, Vol. 89, pp. 33-47.

Bimbato, A.M., 2008, "Analysis of Moving Ground Effects on Aerodynamics Loads of a Body", M.Sc. Dissertation, Mechanical Engineering Institute, UNIFEI, Itajubá, MG, Brazil (in Portuguese).

Blevins, R.D., 1984, "Applied fluid dynamics handbook", Van Nostrand Reinhold Co.

Buresti, G. and Lanciotti, A., 1979, "Vortex Shedding from Smooth and Roghened Cylinders in Cross-Flow near a Plane Surface", Aeronautical Quarterly, Vol. 30, pp. 305-321.

Chorin, A.J., 1973, "Numerical Study of Slightly Viscous Flow", Journal of Fluid Mechanics, Vol. 57, pp. 785-796.

Gerrard, J.H., 1966, "The Mechanics of the Formation Region of Vortices behind Bluff Bodies", J. Fluid Mech., Vol. 25, pp. 401-413.

Grass, A.J., Raven, P.W.J., Stuart, R.J. and Bray, J.A., 1984, "Influence of Boundary Layer Velocity Gradients and Bed Proximity on Vortex Shedding from Free Spanning Pipelines", Transactions of the ASME: Journal of Energy Resources Technology, Vol. 106, pp. 70-78.

Kamemoto, K., 1993, "Procedure to Estimate Unstead Pressure Distribution for Vortex Method" (In Japanese), Trans. Jpn. Soc. Mech. Eng., Vol. 59, 568 B, pp. 3708-3713.

Kamemoto, K., 2004, "On Contribution of Advanced Vortex Element Methods Toward Virtual Reality of Unsteady Vortical Flows in the New Generation of CFD”, J. of the Braz. Soc. of Mech. Sci. \& Eng., Vol. 26, № 4, pp. 368-378.

Katz, J. and Plotkin, A., 1991, Low Speed Aerodynamics: From Wing Theory to Panel Methods. McGraw Hill, Inc.

Leonard, A., 1980, "Vortex Methods for Flow Simulation", J.Comp. Phys., Vol. 37, pp. 89-335.

Lewis, R.I., 1999,"Vortex Element Methods, The Most Natural Approach to Flow Simulation - A Review of Methodology with Applications", Proc. of 1st Int. Conf. on Vortex Methods, Kobe, Nov. 4-5, pp. 1-15.
Lin, C., Lin, W.J. and Lin, S.S., 2005, "Flow Characteristics around a Circular Cylinder near a Plane Boundary", Proceedings of the Sixteenth International Symposium on Transport Phenomena (ISTP-16), 29 August - 1 September, Prague, Czech Republic, (CD-ROOM).

Moura, W.H., 2007, "Analysis of the Flow around an Oscillating Body in the Presence of a Ground Plane" M.Sc. Dissertation, Mechanical Engineering Institute, UNIFEI, Itajubá, MG, Brasil (in Portuguese).

Mustto, A.A., Hirata, M.H. and Bodstein, G.C.R., 1998, "Discrete Vortex Method Simulation of the Flow around a Circular Cylinder with and without Rotation". A.I.A.A. Paper 98-2409, Proceedings of the 16th A.I.A.A. Applied Aerodynamics Conference, Albuquerque, NM, USA, June.

Nishino, T., 2007, "Dynamics and Stability of Flow Past a Circular Cylinder in Ground Effect", Ph.D. Thesis, Faculty of Enginnering, Science and Mathematics, University of Southampton, UK.

Price, S.J., Summer, D., Smith, J.G., Leong, K. and Paidoussis, M.P., 2002, "Flow Vizualization around a Circular Cylinder near to a Plane Wall", Journal of Fluids and Structures, Vol. 16, pp. 175-191.

Ricci, J.E.R., 2002, Numerical Simulation of the Flow Around a Body in the Vicinity of a Plane Using Vortex Method, Ph.D. Thesis, Mechanical Engineering Institute, UNIFEI, Itajubá, MG, Brazil (in Portuguese).

Roshko, A., Steinolfson, A. and Chattoorgoon, V., 1975, "Flow Forces on a Cylinder near a Wall or near Another Cylinder", Proceedings of the 2nd US Nation Conference on Wind Engineering Research, Fort Collins, Paper IV-15.

Sarpkaya, T., 1989, "Computational Methods with Vortices - The 1988 Freeman Scholar Lecture", Journal of Fluids Engineering, Vol. 111, pp.5-52.

Shintani, M. and Akamatsu, T., 1994, "Investigation of Two Dimensional Discrete Vortex Method with Viscous Diffusion Model", International Society of CFD/CFD Journal, Vol. 3, No. 2, pp. 237-254.

Son, J.S. and Hanratty, T.J., 1969, "Velocity Gradients at the Wall for Flow around a Cylinder at Reynolds Number from $5,0 \times 10^{3}$ to $1,0 \times 10^{5}$ ", J. Fluid Mech., Vol. 35, No. 2, pp. 353-368.

Stock, M.J., 2007, "Summary of Vortex Methods Literature (A lifting document rife with opinion)", April, 18: ( 2002-2007 Mark J. Stock.

Taneda, S., 1965, "Experimental Investigation of Vortex Streets", Journal of the Physical Society of Japan, Vol. 20, pp. 1714-1721.

Uhlman, J.S., 1992, "An Integral Equation Formulation of the Equation of an Incompressible Fluid", Naval Undersea Warfare Center, T.R. 10-086.

Zdravkovich, M.M., 1985a, "Observation of Vortex Shedding behind a Towed near a Wall", Flow Visualization III: Proceedings of the Third International Symposium on Flow Vizualization, ed. W.J. Yang, Hemisphere, Washington DC, 423-427.

Zdravkovich, M.M., 1985b, "Forces on a Circular Cylinder near a Plane Wall", Applied Ocean Research, Vol. 7, pp. 197-201.

Zdravkovich, M.M., 2003, "Flow around Circular Cylinders" Vol. 2: Applications, Oxford University Press, Oxford, UK. 